\title{
PREFACE: NON-CLASSICAL MODELS OF AUTOMATA AND APPLICATIONS VIII
}

\author{
Henning Bordihn ${ }^{1, *}$, BenedeK NAGY ${ }^{2,3}$ AND GyÖrgy VASZIL ${ }^{3}$
}

Accepted November 21, 2018.

Several non-classical models of automata and grammar-like structures are studied from different points of view in various areas of theoretical computer science, both as theoretical concepts and as formal models for applications. The purpose of the NCMA workshop series is to promote a deeper and interdisciplinary coverage of this particular area, and to foster new insights and substantial progress in computer science as a whole.

The Eighth Workshop on Non-Classical Models of Automata and Applications (NCMA 2016) was organized by the Department of Computer Science of the Faculty of Informatics at the University of Debrecen, Hungary, August 29-30, 2016. The aim of the workshop was to provide an opportunity for researchers who work on different aspects of non-classical and classical models of automata and grammars to exchange and develop novel ideas.

The history of the NCMA series goes back to 2009 when the first workshop on Non-Classical Models of Automata and Applications was held in Wroclaw, Poland, as a satellite event of the 17th International Symposium on Fundamentals of Computation Theory (FCT 2009). It was sponsored by the AutoMathA project of the European Science Foundation (ESF). The second workshop, NCMA 2010, was held in Jena, Germany, as an associated workshop of the 11th International Conference on Membrane Computing (CMC 11); the third workshop, NCMA 2011, was organized at the University of Milan, Italy, in close proximity to the 15th Conference on Developments in Language Theory (DLT 2011). After 2011, the NCMA workshops were organized as stand-alone events: NCMA 2012 in Fribourg, Switzerland, in August 2012; NCMA 2013 in Umeå, Sweden, in August 2013; NCMA 2014 in Kassel, Germany, in July 2014; and NCMA 2015 in Porto, Portugal, in August 31-September 1, 2015.

In addition to the four invited contributions, papers were submitted to NCMA 2016 by a total of 39 authors, from 18 different countries. On the basis of at least three referees' reports each, the Program Committee selected 15 contributions for presentation. We thank the members of the Program Committee for their excellent work in making this selection:

\footnotetext{
${ }^{1}$ Department of Computer Science, University of Potsdam, August-Bebel-Str. 89, 14482 Potsdam, Germany.

2 Department of Mathematics, Faculty of Arts and Sciences, Eastern Mediterranean University, Famagusta, North-Cyprus, Mersin-10, Turkey.

3 Department of Computer Science, Faculty of Informatics, University of Debrecen, Kassai út 26, 4028 Debrecen, Hungary.

* Corresponding author: henning@cs.uni-potsdam.de
} 
Suna Bensch (Umeå University, Sweden), Henning Bordihn (University of Potsdam, Germany), Erzsébet Csuhaj-Varjú (Eötvös Loránd University, Budapest, Hungary), Alberto Dennunzio (University of MilanoBicocca, Italy), Rudolf Freund (TU Wien, Austria), Violetta Lonati (University of Milan, Italy), Christos Kapoutsis (Carnegie Mellon University, Doha, Qatar), Andreas Maletti (University of Stuttgart, Germany), Alexander Meduna (Brno University of Technology, Czech Republic), Victor Mitrana (University of Bucharest, Romania \& Polytechnic University of Madrid, Spain), Nelma Moreira (University of Porto, Portugal), František Mráz (Charles University in Prague, Czech Republic), Benedek Nagy (University of Debrecen, Hungary \& Eastern Mediterranean University, Famagusta, Cyprus), Linqiang Pan (Huazhong University of Science and Technology, Wuhan, China), Giovanni Pighizzini (University of Milan, Italy), Rogério Reis (University of Porto, Portugal), Bianca Truthe (University of Giessen, Germany), György Vaszil (University of Debrecen, Hungary).

In addition to the invited talks and the regular contributions, NCMA 2016 also featured 7 short presentations to emphasize its workshop character.

We are grateful to the Faculty of Informatics of the University of Debrecen for the financial support of NCMA 2016, and the Organizing Committee, Ildikó Cserhátiné Vecsei and Ernőné Kása, for the local organization. We would also like to thank Rudolf Freund, Sergiu Ivanov, and the Institute of Computer Languages of the TU Wien for their assistance in editing the proceedings which appeared as volume 321 in the series "books@ocg.at" of the Austrian Computer Society, and in editing the booklet containing the short papers.

The authors of selected papers were invited to submit substantially enhanced versions of their contributions to this special issue. We are grateful to all of them for their contributions, and to all the referees for their reports and for their efforts concerning the evaluation of the submissions. We would also like to express our thanks to Christian Choffrut, Sylvain Perifel, and Olivier Serre, Editors-in-Chief of this journal for their support, and to Anne Ruimy and Muriel Bouquant at EDP Sciences for their assistance in realizing this special issue. 